\title{
Rigidity dependence of the intensity variations of galactic cosmic rays
}

\author{
H.Kojima ${ }^{* a}$, S.Shibata $^{b}$, A.Oshima $^{b}$, Y.Hayashi $^{c}$, S.Kawakami $^{c}$, I.Morishita $^{d}$, \\ T.Nakamura $^{e}$, T.Nonaka ${ }^{f}$, S.Ogio ${ }^{c}$, H.Takamaru ${ }^{b}$, K.Tanaka $^{g}$, N.Ito $^{c}$, T.Matsuyama $^{c}$, \\ K.Yamazaki ${ }^{c}$, M.Tokumaru $^{h}$, S.K.Gupta ${ }^{i}$, H.M.Antia ${ }^{i}$, S.R.Dugad ${ }^{i}$, P.K.Mohanty ${ }^{i}$, \\ A.Jain ${ }^{i}$, B.S.Rao ${ }^{i}$ \\ ${ }^{a}$ Faculty of Engineering, Aichi Institute of Technology, Toyota, Aichi 470-0392, Japan \\ ${ }^{b}$ Faculty of Engineering, Chubu University, Kasugai, Aichi 487-8501, Japan \\ ${ }^{c}$ Graduate School of Science, Osaka City University, Osaka 558-8585, Japan \\ ${ }^{d}$ School of Health Sciences, Asahi University, Mizuho, Gifu 501-0296, Japan \\ ${ }^{e}$ Faculty of Science, Kochi University, Kochi 780-8520, Japan \\ ${ }^{f}$ Institute for Cosmic Ray Research, University of Tokyo, Kashiwa, Chiba 277-8582, Japan \\ ${ }^{g}$ Graduate School of Information Sciences, Hiroshima City University, Hiroshima 731-3194, \\ Japan \\ ${ }^{h}$ Solar-Terrestrial Environment Laboratory, Nagoya University Furo-cho, Aichi, Japan \\ ${ }^{i}$ Tata Institute of Fundamental Research, Mumbai, India \\ E-mail: hkojima@aitech.ac.jp
}

\begin{abstract}
For the investigation of the interactions of galactic cosmic rays with the solar wind plasma and/or interplanetary magnetic field, it is important to know the rigidity dependence of the intensity variations of galactic cosmic rays in detail. In this paper, we have performed a regression analysis between the cosmic ray intensity variations and the relative solar wind velocity and obtained the rigidity dependence of the resultant regression coefficients. We have divided the data into two durations of active and calm by a criteria which is based on the data of neutron monitor of the lowest geomagnetic cut-off rigidity on the Earth.
\end{abstract}

The 34th International Cosmic Ray Conference,

30 July- 6 August, 2015

The Hague, The Netherlands

\footnotetext{
* Speaker.
} 


\section{Introduction}

To understand the mechanism of propagation of the galactic cosmic rays in the universe, detail physical process of interactions of the galactic cosmic rays with the interplanetary magnetic field have to be studied. The study on the cosmic ray propagation have been performed mainly focused on the cosmic ray intensity and its anisotropy in the heliosphere. The knowledge of this historic study of the cosmic ray propagation has been expanded its application to the region of an extra heliospheric, namely interstellar space. We know that there are several kinds of anisotropy that vary timewise and spacewise in different scale. Such variations might be observed as results of any effects on the cosmic ray propagation.

It's not obvious how such basic physical mechanisms which could control the cosmic ray propagation behave in different scale. To make this question clear, we investigated the mechanism of cosmic ray intensity variation during two specific periods, namely during so called Forbush Decrease (FDs) as "Active period" and other periods as "Calm period", and made comparison each other. Since it is difficult to dissociate the anisotropy during the FDs, we adopted the solar wind velocity and the cosmic ray intensity as suitable parameters both are less affected by the directional variation.

\section{Observational sites and median rigidities}

In the present analysis, the data obtained by the worldwide network of neutron monitors (NM) [4] and the data from the large area $560 \mathrm{~m}^{2}$ tracking muon telescope of the GRAPES-3 experiment [5] located at Ooty, India were used. The 17 NM stations are listed with their corresponding median primary rigidities $\left(R_{\mathrm{m}}=10.0 \sim 31.6 \mathrm{GV}\right)$, which were calculated from the Tables provided by Yasue and co-workers [6], below in Table.1.

The GRAPES-3 muon telescope consists of 4 super-modules, each in turn having 4 modules. Each module [5] consists of a total of 232 proportional counters (PRCs) arranged in 4 layers, with alternate layers separated by $15 \mathrm{~cm}$ thick concrete and placed in orthogonal directions. One module has a sensitive area of $35 \mathrm{~m}^{2}$ and has the energy threshold of $1 \mathrm{GeV}$ for vertical muons by means of a total of 15 layers of concrete blocks (total thickness $\sim 550 \mathrm{~g} . \mathrm{cm}^{-2}$ ) above the top Layer. This configuration enable us to use the muon detector as telescope. The 9 directional components of large area tracking muon telescope in the GRAPES-3 experiment and the corresponding median primary rigidities $\left(R_{\mathrm{m}}=64.4 \sim 92.0 \mathrm{GV}\right)$ calculated by Nonaka and co-workers [7] are also listed in Table.2.

\section{Selection of Forbush decrease events}

In this paper, we have divided the data into two durations of Active period and Calm period. Active period is only the period of during Forbush Decrease and remaining period after excluding all possible FDs is defined as Calm period. The daily averaged rates from February 2000 to October 2006 observed by the NM stations and the GRAPES-3 muon telescope were used in this analysis. To define the Active period, the following criteria was applied for the FD selection and totally 
Table 1: List of NM stations and corresponding median primary rigidities ( $R_{\mathrm{m}}$ in $\left.\mathrm{GV}\right)$.

\begin{tabular}{|l|c|}
\hline NM station & $R_{\mathrm{m}}(\mathrm{GV})$ \\
\hline Alma-Ata & 15.8 \\
\hline Apatity & 12.6 \\
\hline Athens & 25.1 \\
\hline Beijing & 25.1 \\
\hline Haleakala & 31.6 \\
\hline Inuvik & 12.6 \\
\hline Kiel & 15.8 \\
\hline Lomnicky Stit & 12.6 \\
\hline McMurdo & 12.6 \\
\hline Mexico & 25.1 \\
\hline Moscow & 15.8 \\
\hline Novosibirsk & 15.8 \\
\hline Potchefstroom & 20.0 \\
\hline SouthPole & 10.0 \\
\hline Tbilisi & 20.0 \\
\hline Thule & 12.6 \\
\hline Yakutsk & 12.6 \\
\hline
\end{tabular}

Table 2: List of directional components of GRAPES-3 muon telescope and corresponding median primary rigidities $\left(R_{\mathrm{m}}\right.$ in $\left.\mathrm{GV}\right)$.

\begin{tabular}{|l|c|}
\hline Direction & $R_{\mathrm{m}}(\mathrm{GV})$ \\
\hline $\mathrm{NW}$ & 73.2 \\
\hline $\mathrm{N}$ & 73.5 \\
\hline $\mathrm{NE}$ & 92.0 \\
\hline $\mathrm{W}$ & 64.4 \\
\hline $\mathrm{V}$ & 66.3 \\
\hline $\mathrm{E}$ & 82.9 \\
\hline SW & 70.0 \\
\hline S & 69.9 \\
\hline SE & 88.7 \\
\hline
\end{tabular}

36 FD events which are listed in Table. 3 have been passed the selection filter. All the Active period used in this analysis is defined as a period of 10 days including 3 days in advance of a FD starts.

For the averaged cosmic ray intensity variation with McMurdo and Thule NM stations, if the variation shows $>3 \%$ decrease when compared to the average counting rate of the preceding three days, we define the decrease as a FD event. 
Table 3: List of date of Forbush decreases and their Date.

\begin{tabular}{|l|l|l|l|}
\hline No. & Date of FDs & No. & Date of FDs \\
\hline 1 & 2000.02 .12 & 19 & 2002.07 .20 \\
\hline 2 & 2000.06 .09 & 20 & 2003.05 .30 \\
\hline 3 & 2000.07 .15 & 21 & 2003.10 .22 \\
\hline 4 & 2000.09 .18 & 22 & 2003.10 .30 \\
\hline 5 & 2000.11 .07 & 23 & 2004.01 .22 \\
\hline 6 & 2000.11 .27 & 24 & 2004.07 .24 \\
\hline 7 & 2000.03 .28 & 25 & 2004.07 .27 \\
\hline 8 & 2001.04 .09 & 26 & 2004.09 .14 \\
\hline 9 & 2001.04 .12 & 27 & 2004.11 .08 \\
\hline 10 & 2001.04 .29 & 28 & 2005.01 .03 \\
\hline 11 & 2001.08 .18 & 29 & 2005.01 .18 \\
\hline 12 & 2001.08 .28 & 30 & 2005.05 .09 \\
\hline 13 & 2001.09 .26 & 31 & 2005.05 .15 \\
\hline 14 & 2001.10 .12 & 32 & 2005.07 .17 \\
\hline 15 & 2001.10 .22 & 33 & 2005.08 .24 \\
\hline 16 & 2001.11 .06 & 34 & 2005.09 .11 \\
\hline 17 & 2001.11 .24 & 35 & 2006.12 .08 \\
\hline 18 & 2001.12 .31 & 36 & 2006.12 .15 \\
\hline
\end{tabular}

\section{Solar wind velocity and cosmic ray intensity variation}

The effects of solar wind velocity on the cosmic ray intensity must be through interactions between the interplanetary magnetic field which is frozen in the solar wind and the galactic cosmic ray particles, so we expected it depends on the rigidity of the galactic cosmic rays through the scattering mean free path or the diffusion coefficients of galactic cosmic rays. To investigate such a possible relationship, we chose solar wind velocity as a feasible parameters. Before studying solar wind velocity and cosmic ray intensity correlation, interference by unrelated periodic and transient phenomena in interplanetary space had to be identified and their contribution minimized. Periodic effects include $27 \mathrm{~d}$ solar rotation, annual, $11 \mathrm{yr}$ solar activity, $22 \mathrm{yr}$ solar magnetic cycle etc. Transient phenomena include FDs that cause irregular, short-term CR changes characterized by a sudden drop in a day and a slow recovery spread over days to weeks. Figure. 1 shows the variation of the daily mean solar wind velocity (in $\mathrm{km} / \mathrm{s}$ ), which had already been applied the 27-day high pass filter, provided by the OMNIweb[9]. Figure.2 shows the variation of cosmic ray intensity observed by the Mowcow neutron monitor and the GRAPES-3 muon telescope.

\section{Analysis}

At first, we have taken a correlation between cosmic ray intensity variation and the solar wind velocity and applied regression analysis to obtain the regression coefficient which is the slope of 


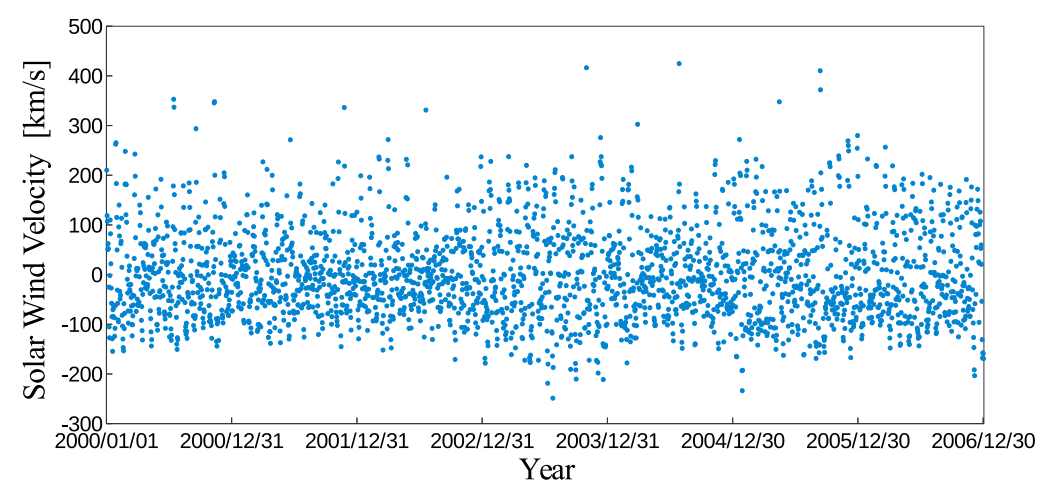

Figure 1: Daily variation in Solar Wind Velocity $V_{S W}$ from 2000 and 2006, provided by OMNIweb.

the straight line fitted onto the scatter plot of data. The regression coefficients, $C_{s w}(\% /(\mathrm{km} / \mathrm{s}))$, between the cosmic ray intensity variations and relative solar wind velocity were obtained for each station. For each station, a scatter plot was drawn using all the daily values of all the events with the cosmic ray intensity variations on the $y$-axis and corresponding relative solar wind velocity on the x-axis respectively. Therefore 26 regression coefficients, $C_{s w}$, were obtained finally. The same procedure has been applied to obtain the regression coefficients $C_{s w, \sigma}(\% /(\mathrm{km} / \mathrm{s}))$ for the correlation between cosmic ray intensity variation and deviation of the solar wind velocity.

We looked into the rigidity dependence of both the regression coefficients $C_{s w}$ and $C_{s w, \sigma}$ as a interested parameters during the Active period and Calm period. $C_{s w}$ shows a relationship between the cosmic ray intensity variation and relative solar wind velocity, $C_{s w, \sigma}$ shows a relationship between the cosmic ray intensity variation and irregularity of relative solar wind velocity.

Using these 26 regression coefficients, the rigidity dependence of them were investigated. A set of $C_{s w}$ s was used to draw a scatter plot using the corresponding median rigidity on the x-axis and applied the further regression analysis to find rigidity dependence as shown in Figure.4. The same procedure was applied to the $C_{s w, \sigma}$ and the rigidity dependence were obtained as shown in Figure.5.

\section{Summary}

For the investigation of the interactions of galactic cosmic rays with the solar wind plasma, it is important to know the rigidity dependence of the intensity variations of galactic cosmic rays in detail. In this paper, we have performed a regression analysis between the cosmic ray intensity variations and the relative solar wind velocity and obtained the rigidity dependence of the resultant regression coefficients. We have investigated the rigidity dependence of regression coefficients, $C_{S w}$ and $C_{s w, \sigma}$, during two specific periods, namely during Forbush Decrease (FDs) as "Active period" and the period after excluding all FDs as "Calm period", We used the World Wide Neutron Monitor stations for the rigidity regions from 10.0 to $31.6 \mathrm{GV}$ and GRAPES-3 muon telescope from 64.4 to $92.0 \mathrm{GV}$. We obtained clear rigidity dependencies in both the Active and Calm periods even in 



Figure 2: Daily variation in Cosmic ray intensity observed by (a) the Moscow neutron monitor and (b) the GRAPES-3 vertical component from 2000 to 2006.

the low and high rigidity regions. This results might help us to understand the mechanism of the interaction of galactic cosmic rays with the solar wind plasma.

\section{Acknowledgment}

We thank all the staff in India for their help in the operation, maintenance of the proportional counters and the associated electronics for the GRAPES-3 tracking muon telescope. We express the special thanks to the GSFC/SPDF OMNIWeb interface enabling us to determine the IMF magnitudes and solar wind velocity. The neutron intensity data used in this work was provided by the World Wide Center(WDC) for Cosmic Rays. This work is supported by the grants-in-aid of the Solar-Terrestrial Environment Laboratory of Nagoya University and the Institute for Cosmic Ray Research of University of Tokyo, Chubu University and National Astronomical Observatory of Japan.

\section{References}

[1] H.V.Cane: Coronal mass ejections and forbush decreases. Space Sci. Revs. 93, 55-77 (2000)

[2] J.A.Lockwood: Forbush decreases in the cosmic radiation. Space Sci. Revs. 12, 658-715 (1971) 


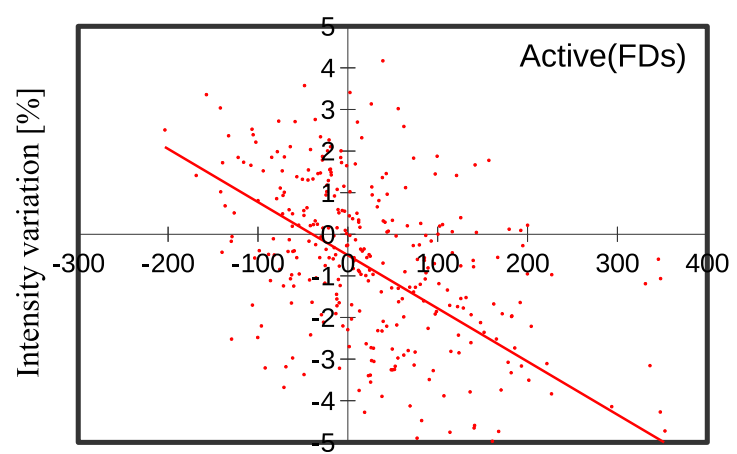

Solar Wind Velocity $[\mathrm{km} / \mathrm{s}]$

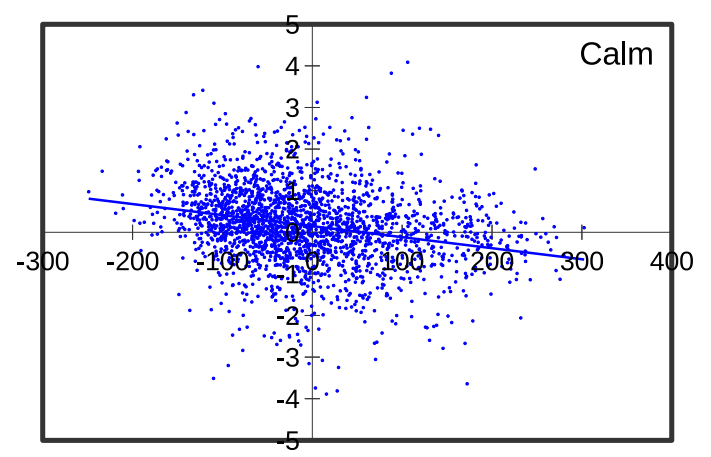

Solar Wind Velocity $[\mathrm{km} / \mathrm{s}]$

Figure 3: The relationship between cosmic ray intensity variation and the relative solar wind velocity observed by Moscow neutron monitor. The slope of the solid line in both the figures show a regression coefficient, $C_{s w}$ in $\% /(\mathrm{km} / \mathrm{s})$, for (a) Active period and (b) Calm period.
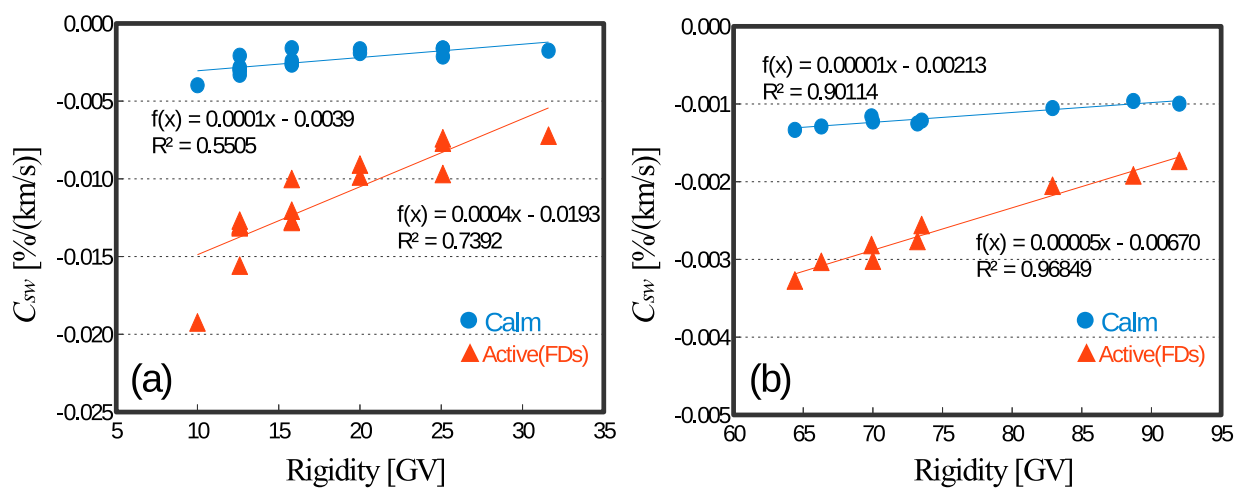

Figure 4: Rigidity dependence of the regression coefficient $C_{S w}$ in $\% /(\mathrm{km} / \mathrm{s})$; (a) Worldwide network of Neutron Monitors, (b) GRAPES-3 Muon telescope

[3] S.Sakakibara, K.Nagashima: Rigidity spectrum of forbush decrease. In: Proc. Int. Sympo. on Cosmic Ray Modulation in the Heliosphere,Morioka, pp. 212-218 (1984). ICRC

[4] Network of Cosmic Ray Stations, RFBR, Russia. http://cr0.izmiran.rssi.ru/common/links.htm

[5] Y.Hayashi et al.: A large area muon tracking detector for ultra-high energy cosmic ray astrophysics the GRAPES-3 experiment. Nucl. Instrum. Methods A 545, 643 (2005)

[6] S.Yasue, S.Mori, S.Sakakibara, K.Nagashima: Coupling Coefficients of Cosmic Ray Daily Variations for Neutron Monitor Stations. Report of Cosmic-Ray Research Laboratory, Nagoya University, No.7 (1982)

[7] T.Nonaka: A study of loss-cone precursor decrease with grapes-3 muon telescopes (in japanese). $\mathrm{PhD}$ thesis, Osaka City University (2006) 

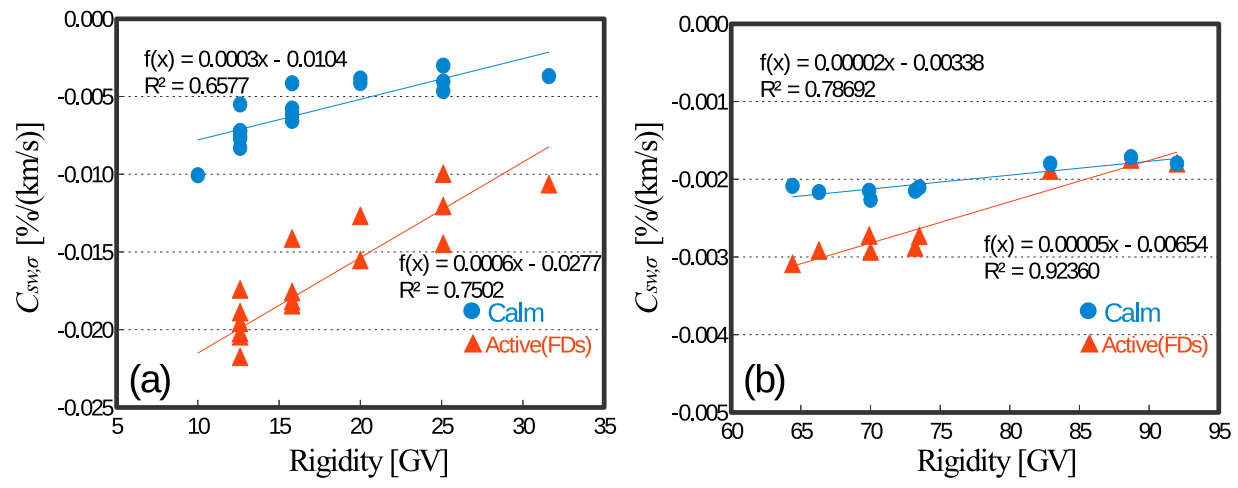

Figure 5: Rigidity dependence of the regression coefficient $C_{s w, \sigma}$ in $\% /(\mathrm{km} / \mathrm{s})$; (a) Worldwide network of Neutron Monitors, (b) GRAPES-3 Muon telescope

[8] SolarSoft. http://www.lmsal.com/solarsoft/

[9] OMNI 2 Preparation. http://omniweb.gsfc.nasa.gov/html/omni2doc.html 